\title{
Single-stranded DNA (ssDNA) Labeling Index Is Related to Risk Grade in Patients with Gastrointestinal Stromal Tumors
}

\author{
KUNIO MOCHIZUKI, TETSUO KONDO, NAOKI OISHI, IPPEI TAHARA, \\ TOMOHIRO INOUE, KAZUNARI KASAI, TADAO NAKAZAWA and RYOHEI KATOH \\ Department of Pathology, School of Medicine, University of Yamanashi, Chuo, Japan
}

\begin{abstract}
Background/Aim: The main objective of the present study was to evaluate the significance of apoptosis in the Fletcher's risk classification for gastrointestinal stromal tumors (GISTS). Materials and Methods: Apoptotic cells were identified by immunostaining for single-stranded DNA (ssDNA). We assigned each GIST to one of four risk groups: very low risk, $n=32$; low risk, $n=53$; intermediate risk, $n=15$; high risk, $n=6$ ). Results: The mean ssDNA labeling index for each group was $8.0 \pm 44.2,20.1 \pm 86.5,18.7 \pm 38.6$ and $5.7 \pm 5.7$, respectively. Fletcher's risk classification for GISTs correlated significantly with the ssDNA labeling index ( $p=0.002)$. Conclusion: The ssDNA labeling index and Ki-67 labeling index were the most significant factors corresponding to the risk grade of GISTs. These findings suggest that the ssDNA labeling index might be useful for predicting aggressive biological behavior of GISTs.
\end{abstract}

Gastrointestinal stromal tumors (GISTs) arise from the interstitial cells of Cajal or a common cellular precursor, both of which express type III tyrosine kinase receptors; mutations in the tyrosine kinase receptor c-KIT (CD117) or related tyrosine kinase receptors contribute to loss of growth control and tumor formation in GISTs (1). GISTs are found most commonly in the stomach $(50-60 \%)$, followed by the small intestine (30-35\%), colon and rectum (5\%), esophagus $(<1 \%)$, and rarely in locations outside the gastrointestinal tract (mesentery, omentum, and retroperitoneum; $<5 \%$ ). The median age at diagnosis is 63 years, and fewer than $1 \%$ of patients are younger than 20 years old (1).

Tumor growth depends on the equilibrium between cell proliferation and cell death. Apoptosis (programmed cell death) is considered an important homeostatic mechanism for

Correspondence to: Kunio Mochizuki, MD, Ph.D., Department of Pathology, School of Medicine, University of Yamanashi, 1110 Shimokato, Chuo, Yamanashi, 409-3898, Japan. Tel: +81 552739529, Fax: +81 552739534, e-mail: kuniom@yamanashi.ac.jp

Key Words: Apoptosis, single-stranded DNA, gastrointestinal stromal tumor, Fletcher's risk classification. balancing cell proliferation and maintaining the correct number of cells under physiological and pathological conditions (2). Terminal deoxynucleotidyl transferase dUTP nick end labeling (TUNEL) is a technique that allows detection of the initial stages of apoptosis (2). However, the TUNEL in situ technique for detecting apoptosis is not completely specific, as overlap between apoptotic and necrotic cell death has been reported, and may result from the fact that some of the apoptotic cells do not stain (3). Single-stranded DNA (ssDNA) labeling is sufficiently sensitive to show apoptosis and is more specific to apoptosis by not staining necrotic cells $(4,5)$.

The apoptotic rate has been shown to correlate with clinical behavior and prognosis of a limited number of solid tumor types (6-9). To our knowledge, there exist few English language publications on the relationship between GISTs and apoptosis $(10,11)$. However, some reports showed a biological relationship between GISTs and other factors: the risk classification for GISTs may be significantly associated with the Ki-67 labeling index, blood vessel invasion (BVI) and microvessel density (MVD) (12-15).

The main objective of this study was to evaluate the significance of apoptosis in the Fletcher's risk classification (16) for GISTs. Because ssDNA seems to be more specific for apoptosis, we used ssDNA immunostaining to identify apoptotic cells. We studied 106 GIST samples using the ssDNA labeling index in conjunction with Ki-67 labeling index, BVI, lymphatic vessel invasion (LVI) and MVD.

\section{Materials and Methods}

Patients. We examined 103 cases of GIST (106 samples: three cases had two GISTs each), using samples obtained surgically between January 2000 and May 2015 at the University of Yamanashi Hospital and between April 2006 and May 2015 at the KofuKyoritsu Hospital. Table I summarizes the clinicopathological findings of the GIST samples. There were 59 men and 47 women, ranging in age from 35-94 years, with a mean age of 69 years. The majority of GISTs were located in the stomach (87 samples). We followed Fletcher's criteria for histological diagnosis and risk classification (16). All GISTs showed consistent immunopositivity 
for c-KIT. GISTs were assigned to one of four risk groups (very low risk, $\mathrm{n}=32$; low risk, $\mathrm{n}=53$; intermediate risk, $\mathrm{n}=15$; high risk, $\mathrm{n}=6$ ). Two pathologists (K.M. and R.K.) independently reviewed hematoxylin and eosin-stained slides blinded to the original pathological diagnosis. The Research Ethics Committee of Faculty of Medicine, University of Yamanashi approved this study (approval number 1639).

Immunohistochemistry. Sections 4- $\mu \mathrm{m}$ thick were cut from formalin-fixed, paraffin-embedded tissue blocks and then dewaxed and rehydrated. Immunohistochemical staining was performed on representative slides. Table II lists the primary antibodies used, their dilutions and their sources. Antigen retrieval was accomplished through heat treatment before performing the primary antibody reactions: autoclaving at $120^{\circ} \mathrm{C}$ for $10 \mathrm{~min}$ in citrate buffer $\mathrm{pH} 6$ for c-KIT and $\mathrm{Ki}-67$, or in Antigen Retrieval Solution pH 9 (Nichirei Biosciences, Tokyo, Japan) for CD31 or citrate buffer $\mathrm{pH} 7$ for podoplanin. After inhibiting endogenous peroxidase, we used positive controls (cKIT: GIST; ssDNA: tonsil; Ki-67: GIST; CD31: vascular endothelium; podoplanin: lymphatic endothelium) to perform the primary antibody reactions. We used the N-Histofine Simple Stain MAX PO (MULTI) (Nichirei Biosciences, Tokyo, Japan) with diaminobenzidine as a chromogen and a light counterstain with hematoxylin to perform immunohistochemistry. K.M. and R.K. simultaneously reviewed immunostained sections using a doubleheaded light microscope.

ssDNA labeling index. K.M. and R.K. assessed apoptotic cells using ssDNA labeling. They considered cells with immunostaining in the nuclei or immunostaining in apoptotic bodies as being positively labeled, and we used the percentage of immunostained cells out 1,000 cells counted in areas of the strongest nuclear labelling ('hot spots') as the ssDNA labeling index (17).

Ki-67 labeling index. We chose the field that had the maximum density of Ki-67-immunopositive nuclei and evaluated the Ki-67 labeling index as proliferative activity by using 'GunmaLI' (18).

Vessel invasion. BVI was defined as the presence of clusters of GIST cells within areas surrounded by elastic fibers of the vessel wall stained with elastica-van Gieson (EVG) stain. During this process, sections 4- $\mu$ m thick were cut from formalin-fixed, paraffinembedded tissue blocks. After deparaffination, they were transferred to $70 \%$ ethanol. They were stained with Weigert's Resorcin Fuchsin solution for 60-120 min and washed in 100\% ethanol. After rinsing, they were stained with iron hematoxylin for $10 \mathrm{~min}$, and washed in running water. After differentiation with $1 \% \mathrm{HCl}$-water, they were stained with van Gieson solution for $5 \mathrm{~min}$. The slides were dehydrated with ethanol series and cleared with xylene. LVI was then defined as the presence of clusters of GIST cells within areas surrounded by lymphatic endothelial cells immunostained for podoplanin (14).

Microvessel density. We evaluated MVD by immunohistochemistry of tumor vessels for CD31. Any single immunopositive cell or cluster of cells clearly separated from adjacent clusters and background, with or without lumen, was considered an individual vessel, and we excluded areas of fibrosis, necrosis and inflammation from the counting (19). The immunostained sections were scanned
Table I. Clinicopathological characteristics of gastrointestinal stromal tumor samples.

\begin{tabular}{lc}
\hline Characteristic & Value \\
\hline Gender, n (\%) & \\
$\quad$ Male & $59(56 \%)$ \\
Female & $47(44 \%)$ \\
Age, years & $69(35-94)$ \\
$\quad$ Mean (range) & \\
Anatomical location & $87(82 \%)$ \\
Stomach & $19(18 \%)$ \\
Small intestine & $32(30 \%)$ \\
Very low & \\
Fletcher's risk classification & $53(50 \%)$ \\
Low & $15(14 \%)$ \\
Intermediate & $6(6 \%)$ \\
High & \\
\hline
\end{tabular}

at low magnification (40x). Then K.M. and R.K. together selected three tumor areas with positive CD31 staining having the highest density of distinctly highlighted microvessels ('hot spots') within each section. They counted the MVD in the three most vascularized areas within a 400× microscopic field of an Olympus BX50 (Tokyo, Japan) microscope (19).

Statistical analysis. We used the Kruskal-Wallis test or Fisher's exact test to evaluate differences between the Fletcher's risk classification for GISTs regarding ssDNA labeling index, Ki-67 labeling index, BVI, LVI and MVD. Differences with a $p$-value of less than 0.05 indicated statistical significance. Statistical analysis was carried out using IBM SPSS Statistics version 22 (IBM Corp., NY, USA)

\section{Results}

Using immunohistochemistry, the means for the ssDNA labeling indices by risk group were $8.0 \pm 44.2$ for very low risk, $20.1 \pm 86.5$ for low risk, $18.7 \pm 38.6$ for intermediate risk and 5.7 \pm 5.7 for high risk GISTs (Figure 1C). The corresponding mean Ki-67 labeling indices obtained through immunohistochemistry were $1.7 \pm 1.6,3.1 \pm 2.4,3.5 \pm 4.5$ and

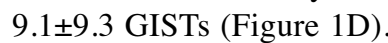

There was BVI in 23 samples (very low risk, $n=2$; low risk, $\mathrm{n}=16$; intermediate risk, $\mathrm{n}=3$; high risk, $\mathrm{n}=2$ ) observed using EVG stain (Figure 1E), and LVI was detected in only one sample using podoplanin immunostaining (Figure 1F).

The means for MVD obtained by CD31 immunostaining were $31.3 \pm 22.3,44.3 \pm 27.1,49.9 \pm 27.4$ and $45.2 \pm 18.2$, respectively (Figure $1 \mathrm{G})$.

Table III summarizes the statistical analyses. Fletcher's risk classification for GISTs correlated significantly with the ssDNA labeling index, Ki-67 labeling index, BVI and the MVD $(p=0.002, p=0.002, p=0.035$ and $p=0.042$, respectively). However, we were unable to demonstrate 

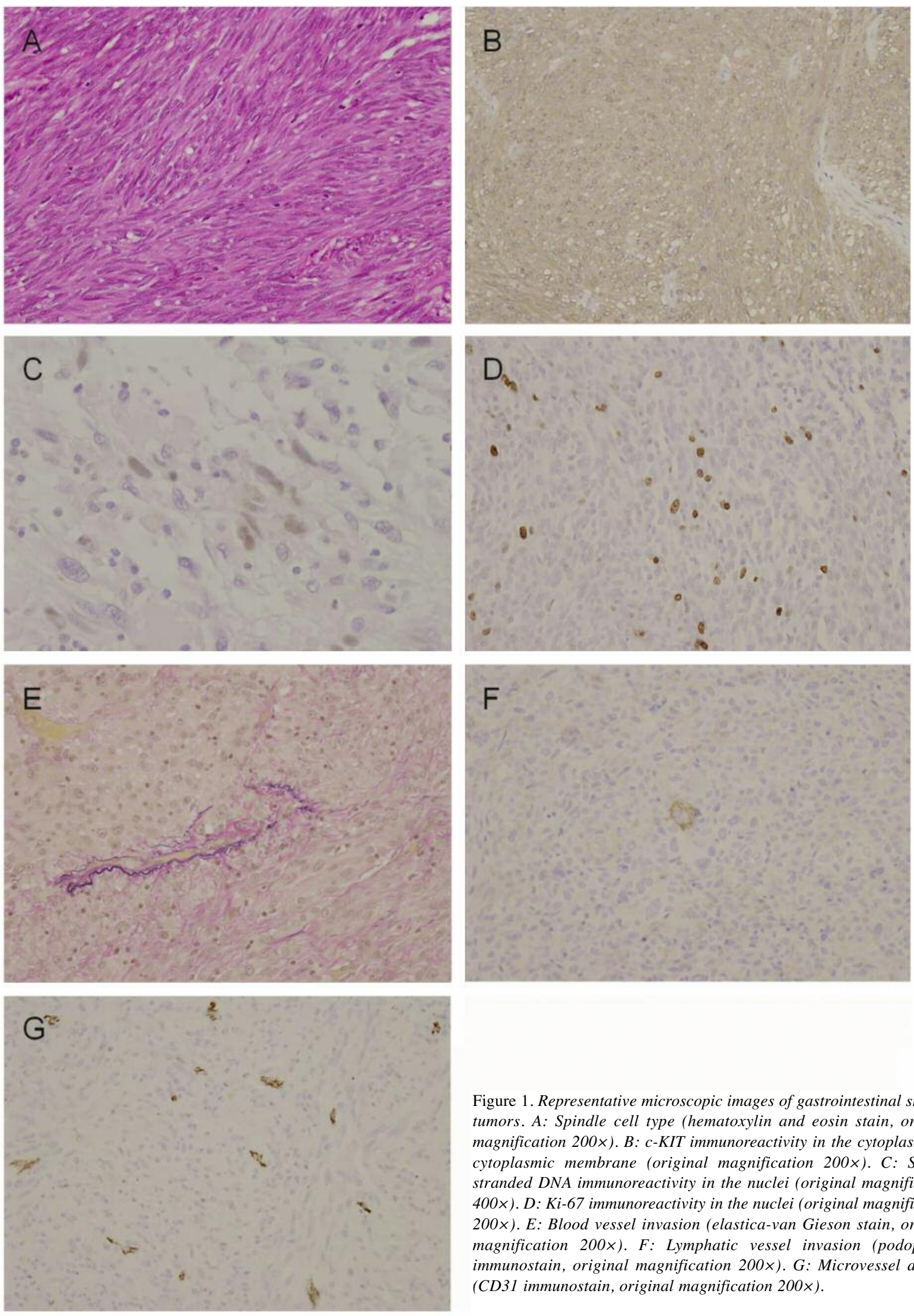

Figure 1. Representative microscopic images of gastrointestinal stromal tumors. A: Spindle cell type (hematoxylin and eosin stain, original magnification 200x). B: c-KIT immunoreactivity in the cytoplasm and cytoplasmic membrane (original magnification 200x). C: Singlestranded DNA immunoreactivity in the nuclei (original magnification 400x). D: Ki-67 immunoreactivity in the nuclei (original magnification 200x). E: Blood vessel invasion (elastica-van Gieson stain, original magnification 200x). F: Lymphatic vessel invasion (podoplanin immunostain, original magnification 200x). G: Microvessel density (CD31 immunostain, original magnification 200x). 
Table II. Antibodies used in this study.

\begin{tabular}{llcc}
\hline Antibody for & & Source & Dilution \\
\hline c-KIT & Rabbit polyclonal & Santa Cruz Biotechnology, TX, USA & $1: 50$ \\
ssDNA & Rabbit polyclonal & Immuno-Biological Laboratories, Gunma, Japan & $1: 200$ \\
Ki-67 & MIB-1 & Dako, Glostrup, Denmark & $1: 100$ \\
CD31 & JC/70A & Dako, Glostrup, Denmark & Pre-diluted \\
Podoplanin & D2-40 & Dako, Glostrup, Denmark & $1: 200$ \\
\hline
\end{tabular}

Table III. Association between Fletcher's risk classification of the gastrointestinal stromal tumors and study variables.

\begin{tabular}{|c|c|c|c|c|c|c|}
\hline \multirow[b]{2}{*}{ Factor } & & \multicolumn{4}{|c|}{ Risk group } & \multirow[b]{2}{*}{$p$-Value } \\
\hline & & Very low $(\mathrm{n}=32)$ & Low $(\mathrm{n}=53)$ & Intermediate $(\mathrm{n}=15)$ & High $(n=6)$ & \\
\hline ssDNA labeling index & Mean \pm SD & $8.0 \pm 44.2$ & $20.1 \pm 86.5$ & $18.7 \pm 38.6$ & $5.7 \pm 5.7$ & 0.002 \\
\hline Ki-67 labeling index & Mean \pm SD & $1.7 \pm 1.6$ & $3.1 \pm 2.4$ & $3.5 \pm 4.5$ & $9.1 \pm 9.3$ & 0.002 \\
\hline \multirow[t]{2}{*}{ Blood vessel invasion } & Absent, n (\%) & $30(94 \%)$ & $37(70 \%)$ & $12(80 \%)$ & $4(67 \%)$ & 0.035 \\
\hline & Present, n (\%) & $2(6 \%)$ & $16(30 \%)$ & $3(20 \%)$ & $2(33 \%)$ & \\
\hline \multirow[t]{2}{*}{ Lymphatic vessel invasion } & Absent, n (\%) & $32(100 \%)$ & $52(98 \%)$ & $15(100 \%)$ & $6(100 \%)$ & $>0.99$ \\
\hline & Present, n (\%) & $0(0 \%)$ & $1(2 \%)$ & $0(0 \%)$ & $0(0 \%)$ & \\
\hline Microvessel density & Mean \pm SD & $31.3 \pm 22.3$ & $44.3 \pm 27.1$ & $49.9 \pm 27.4$ & $45.2 \pm 18.2$ & 0.042 \\
\hline
\end{tabular}

SD: Standard deviation.

statistical significance between Fletcher's risk classification for GISTs and LVI $(p=1.000)$.

\section{Discussion}

Apoptosis is an inborn process preserved during evolution. It allows cells to systematically inactivate, destroy and dispose of their own components thus leading to their death (20). This cycle can be activated by both intra and extracellular mechanisms. The intracellular components involve a genetically defined development process, while the extracellular aspects depend on endogenous proteins, cytokines and hormones, as well as xenobiotics, radiation, oxidative stress and hypoxia (20). The apoptotic GIST cells (ssDNA-labeled cells) were situated locally and together in the tumor, frequently around hemorrhagic areas (ischemic areas). Furthermore, we found that a higher ssDNA labeling index correlated significantly with Fletcher's higher risk GIST ( $p=0.002$ ). Therefore, our results suggest that hypoxia induced by ischemia may induce apoptosis of the GIST cells, and ischemic conditions may be more prevalent in higher risk GISTs than in lower risk GISTs. On the other hand, Cunningham et al. showed that apoptosis in GISTs was not significantly associated with tumor size and mitotic index (10), and Wang et al. indicated that the apoptotic index gradually decreased in specimens from patients with benign
GISTs, potentially malignant GISTs and malignant GISTs, as demonstrated by TUNEL (11). The discrepancy between our results and their results may be due to the different determination methods used (ssDNA immunostaining vs TUNEL) for identifying GIST cells in a state of apoptosis.

We also studied the biological relationships between GISTs and other factors and found that the Fletcher's risk classification for GISTs was significantly associated with the Ki-67 labeling index $(p=0.002)$, BVI $(p=0.035)$ and MVD $(p=0.042)$. We were unable to demonstrate statistical significance of association between Fletcher's risk classification for GISTs and LVI $(p>0.99)$. This is almost identical to previous reports (12-15).

In conclusion, the ssDNA labeling index and the Ki-67 labeling index were the most significant factors associated with the risk grade of GISTs. These findings suggest that the ssDNA labeling index might be useful for predicting aggressive biological behavior of GISTs. Nevertheless, the number of intermediate- and high-risk case was not high in this study. Further studies of more intermediate- and highrisk cases are needed to determine the accurate usefulness of ssDNA labeling index as a biomarker for GIST.

\section{Conflicts of Interest}

None of the Authors have any conflict of interest in regard to this study. 


\section{References}

1 Valsangkar N, Sehdev A, Misra S, Zimmers TA, O’Neil BH and Koniaris LG: Current management of gastrointestinal stromal tumors: Surgery, current biomarkers, mutations, and therapy. Surgery 158: 1149-1164, 2015.

2 Sirvent JJ, Aguilar MC, Olona M, Pelegrí A, Blázquez S and Gutiérrez C: Prognostic value of apoptosis in breast cancer (pT1pT2). A TUNEL, p53, BCL-2, BAG-1 and BAX immunohistochemical study. Histol Histopathol 19: 759-770, 2004.

3 Ben-Izhak O, Laster Z, Araidy S and Nagler RM: TUNEL - an efficient prognosis predictor of salivary malignancies. $\mathrm{Br} \mathrm{J}$ Cancer 96: 1101-1106, 2007.

4 Grasl-Kraupp B, Ruttkay-Nedecky B, Koudelka H, Bukowska $\mathrm{K}$, Bursch $\mathrm{W}$ and Schulte-Hermann R: In situ detection of fragmented DNA (TUNEL assay) fails to discriminate among apoptosis, necrosis, and autolytic cell death: a cautionary note. Hepatology 21: 1465-1468, 1995.

5 Frankfurt OS, Robb JA, Sugarbaker EV and Villa L: Monoclonal antibody to single-stranded DNA is a specific and sensitive cellular marker of apoptosis. Exp Cell Res 226: 387-397, 1996.

6 Aihara M, Scardino PT, Truong LD, Wheeler TM, Goad JR, Yang $G$ and Thompson TC: The frequency of apoptosis correlates with the prognosis of Gleason grade 3 adenocarcinoma of the prostate. Cancer 75: 522-529, 1995.

7 Lipponen PK and Aaltomaa S: Apoptosis in bladder cancer as related to standard prognostic factors and prognosis. J Pathol 173: 333-339, 1994.

8 Lipponen P, Aaltomaa S, Kosma VM and Syrjänen K: Apoptosis in breast cancer as related to histopathological characteristics and prognosis. Eur J Cancer 30A: 2068-2073, 1994.

9 Törmänen U, Eerola AK, Rainio P, Vähäkangas K, Soini Y, Sormunen R, Bloigu R, Lehto VP and Pääkkö P: Enhanced apoptosis predicts shortened survival in non-small cell lung carcinoma. Cancer Res 55: 5595-5602, 1995.

10 Cunningham RE, Abbondanzo SL, Chu WS, Emory TS, Sobin LH, O'Leary TJ: Apoptosis, bcl-2 expression, and p53 expression in gastrointestinal stromal/smooth muscle tumors. Appl Immunohistochem Mol Morphol 9: 19-23, 2001.

11 Wang Q and Kou YW: Study of the expressions of $p 53$ and $B C L-2$ genes, the telomerase activity and apoptosis in GIST patients. World J Gastroenterol 13: 2626-2628, 2007.
12 Nakamura N, Yamamoto H, Yao T, Oda Y, Nishiyama K, Imamura M, Yamada $\mathrm{T}$, Nawata $\mathrm{H}$ and Tsuneyoshi $\mathrm{M}$ : Prognostic significance of expressions of cell-cycle regulatory proteins in gastrointestinal stromal tumor and the relevance of the risk grade. Hum Pathol 36: 828-837, 2005.

13 Huang HY, Huang WW, Lin CN, Eng HL, Li SH, Li CF, Lu D, Yu SC and Hsiung CY: Immunohistochemical expression of p16INK4A, Ki-67, and MCM2 proteins in gastrointestinal stromal tumors: prognostic implications and correlations with risk stratification of NIH consensus criteria. Ann Surg Oncol 13: 1633-1644, 2006.

14 Yamamoto H, Kojima A, Miyasaka Y, Imamura M, Nakamura N, Yao T, Tsuneyoshi M and Oda Y: Prognostic impact of blood vessel invasion in gastrointestinal stromal tumor of the stomach. Hum Pathol 41: 1422-1430, 2010.

15 Imamura M, Yamamoto H, Nakamura N, Oda Y, Yao T, Kakeji $\mathrm{Y}$, Baba H, Maehara $\mathrm{Y}$ and Tsuneyoshi M: Prognostic significance of angiogenesis in gastrointestinal stromal tumor. Mod Pathol 20: 529-537, 2007.

16 Fletcher CD, Berman JJ, Corless C, Gorstein F, Lasota J, Longley BJ, Miettinen M, O'Leary TJ, Remotti H, Rubin BP, Shmookler B, Sobin LH and Weiss SW: Diagnosis of gastrointestinal stromal tumors: A consensus approach. Hum Pathol 33: 459-465, 2002.

17 Morinaga S, Ishiwa N, Noguchi Y, Yamamoto Y, Rino Y, Imada T, Takanashi Y, Akaike M, Sugimasa Y and Takemiya S: Growth index, assessed with Ki-67 and ssDNA labeling; a significant prognosticator for patients undergoing curative resection for hepatocellular carcinoma. J Surg Oncol 92: 331-336, 2005.

18 Tanaka G and Nakazato Y: Automatic quantification of the MIB1 immunoreactivity in brain tumors. International Congress Series 1259: 15-19, 2004.

19 Lopes N, Sousa B, Vieira D, Milanezi F and Schmitt F: Vessel density assessed by endoglin expression in breast carcinomas with different expression profiles. Histopathology 55: 594-599, 2009.

20 Stoian M, State N, Stoica V and Radulian G: Apoptosis in colorectal cancer. J Med Life 7: 160-164, 2014.

Received February 22, 2018

Revised March 15, 2018

Accepted March 23, 2018 